\title{
Role of Endovascular Recanalization and Stenting of Total Occlusions of The Renal Arteries For Blood Pressure Control in Resistant to Treatment Hypertension
}

\author{
Ivo Petrov, Iveta Tasheva, *Iskren Garvanski, Iana Simova, Mihail Marzyanov and Gloria Adam \\ City Clinic University Hospital, Bulgaria
}

Submission: December 14, 2016; Published: January 03, 2017

*Corresponding author: Iskren Garvanski, City Clinic University Hospital, Bulgaria Sofia str. Okolovrasten pat 127, Tel: +359885933752; Email: i.garvanski@gmail.com

\begin{abstract}
Purpose: To assess $\mathrm{t}$ ! he effect on blood pressure (BP) control and rennin levels of the percutaneous recanalization of totally occluded renal arteries in patients with resistant arterial hypertension, preserved blood flow in the sub-segmental renal arteries, and high level of plasma rennin.

Methods: Between 2011 and 2015, we examined 7 patients with total occlusion of a Percutaneous recanalization was attempted in all of them. Success was achieved in $6(85.7 \%)$ of the cases. All patients were hypertensive before the procedure, with mean BP values of $167.1 / 95.1$ mmHg under systematic antihypertensive treatment with at least 3 antihypertensive agents. In all patients, plasma rennin activity levels were more than $2,5 \mathrm{ng} / \mathrm{mL} / \mathrm{h}$ before the procedure. The patients had duplex signs of occluded renal artery and partially preserved subsegmental flow. Two of the patients showed normal serum creatinine levels, and one of them had CKD on chroniodialysis. The other three patients had slightly increased creatinine levels, and in two of them, creatinine levels normalized in the first follow-up month, and in the remaining patient, there was no significant change after the procedure. For recanalization of the occlusions of the renal arteries, we used coronary CTO techniques.
\end{abstract}

Results: Percutaneous recanalization of renal CTO was attempted in seven patients and was successful in six of the cases. Clinical and Duplex follow-up was performed at 4 weeks, 3 months, 6 months, and 1 year after the intervention. BP was significantly reduced in all of the patients who had undergone successful revascularization. Two cases of in stent restenosis showed increased BP levels, which normalized again after the second PTA. In all of the patients with successful procedure, normal rennin levels were established at 6 months and 1 year.

Conclusion: In cases of total renal artery occlusion, the most probable mechanism of resistant AH is the preserved microcirculation allowing juxtaglomerular cells survival resulting in elevated renin production. In case of renal occlusion and resistant AH, preserved renin production is a probable predictor of clinical success after recanalization. At the same time, preserved microcircular perfusion is a predictor of renin-lowering effect and blood-pressure-control success after opening a renal CTO. There is evidence of a relationship between preserved parenchymal flow and the expected postinterventional result regarding BP control, confirmed in our cases. Endovascular recanalization of total renal artery occlusion is feasible and safe. This procedure has to be applied only to a selective group of patients with resistant hypertension and evidence of preserved subsegmental flow.

Abbreviations: BP: Blood Pressure ; RAS: Renal Artery Stenosis ; FMB: Fibro Muscular Dysplasia ; CTO: Chronic Total Occlusions ; ABPM: Ambulatory Blood Pressure Monitoring

\section{Introduction}

Hypertension affects more than $25 \%$ of the worldwide adult population [1]. Although the vast majority of patients suffer from essential hypertension, it is important to identify patients with secondary treatable causes of hypertension, especially renal artery stenosis (RAS), which is the usual cause of hypertension resistant to medical treatment [2]. The two main causes of renal artery stenosis are atherosclerosis and fibro muscular dysplasia (FMB). Atherosclerosis accounts for about 90\% of all cases of RAS, while FMB is the cause of about $10 \%$. FMD is most common in women between 20 and 50 years of age and its progression to total occlusion is rare, compared to that of atherosclerotic renal 
artery stenosis [3]. Atherosclerotic disease of the renal artery, which is frequently responsible for uncontrollable hypertension, congestive heart failure, and progressive renal failure leading to endstage renal disease, is prevalent among elderly patients [4].

The first percutaneous transluminal renal angioplasty (PTRA) for the treatment of atherosclerotic renal artery stenosis was performed by Gruentzig in 1977, the same year as the first coronary angioplasty was performed $[5,6]$. Later on, stunting has emerged as a procedure, associated with low mortality and morbidity for symptomatic renovascular disease. Since then, a lot of clinical data has been gathered, raising controversies about the effect of renal artery stunting in the treatment of renovascular hypertension and chronic renal failure. Further, while stunting of the renal artery stenosis is still justified and widely performed, chronic total occlusions (CTO) of the renal arteries are largely considered inappropriate for endovascular treatment $[7,8]$.

Indeed, in most cases, the total occlusion of a renal artery, supplying a small atrophied kidney may not be considered an appropriate target for intervention. In fact in the largely accepted guidelines, the small size of the target kidney (less than $7 \mathrm{~cm}$ ) is a contraindication for renal artery intervention. However, in case a group of patients with occluded renal arteries, which have the potential to benefit from recanalization and full restoration of flow after balloon PTA or stunting, it is important for them to be identified. There are scarce clinical data for technical feasibility and clinical effect of the Percutaneous recanalization of occluded renal arteries and only several case reports with positive results have been published thus far [9-11].

The purpose of this registry was to test whether Percutaneous recanalization and stunting of totally occluded renal arteries might be justified as effective in reducing the blood pressure (BP) in some patients with chronically occluded renal arteries and to identify specific predictors of clinical effect, which are probably preserved cortical blood flow and high levels of plasma renin.

\section{Materials and Methods}

Inclusion criteria for this pilot registry were as follows:
a. resistance to medical treatment hypertension
b. evidence of totally occluded renal artery
c. high level of plasma renin activity

d. Doppler US evidence of total occlusion of renal artery with preserved cortical and subsegmental blood flow

In the registry, all consecutive patients between January 2011 and May 2015 who fulfilled the inclusion criteria were included. Seven consecutive patients ( 3 women, 4 men) at average age of 42.8 years (range, 15-67 years) met the inclusion criteria and were included in the registry. Percutaneous recanalization of the renal artery occlusion was attempted in all 7 patients. The underlying pathological vascular process was as follows: three of the patients had fibro muscular dysplasia, one had Takayasu arteritis, one had dissection, and two of the patients had atherosclerotic renal artery occlusions.

All 7 patients had severe uncontrolled arterial hypertension, defined as average systolic pressure of $>140 \mathrm{mmHg}$ and average diastolic pressure of $>90 \mathrm{mmHg}$, while on treatment with $\geq 3$ antihypertensive drugs, at least one of which was a diuretic. Average BP values obtained by 24-h ambulatory BP monitoring (ABPM) including on the day before hospital admission were a mean of 167.1/95.1 mmHg. The patients were considered eligible for the procedure according to the two following criteria:

\section{i. Resistant arterial hypertension}

ii. Duplex evidence of total renal artery occlusion

iii. High level of plasma renin activity $>2.5 \mathrm{ng} / \mathrm{mL} / \mathrm{h}$.

iv. Doppler sonography evidence of preserved flow in the sub segmental arteries (interlobular and arcuate arteries)

Technical success was defined as restoration of the ante grade contrast delivery in the sub segmental renal arteries, lack of residual stenosis $>50 \%$ and /or flow limiting dissection.

All patients had Duplex signs of renal artery occlusion: 2 of the left renal arteries and 5 of the right renal arteries, as well as partially preserved sub segmental flow, appeared on color coded Duplex sonography. Three of the patients showed normal serum creatinine levels, three showed CKD not indicated for dialysis treatment, and one was undergoing chroniodialysis.

For treating chronic totally occluded renal arteries, coronary CTO techniques were used. In three cases, the procedure was performed using a 6 Fr right radial approach with a JR 3.5/6 Fr guiding catheter; three with right femoral approach and one with right brachial approach. The unsuccessful procedure was done through femoral approach. In all of the 6 successful cases crossing of the lesion was achieved using CTO dedicated guide wires and the use of a low profile over-the-wire OTW balloon.

Coronary balloons were used for pre dilatation (ranged approximately $1.25-2.0 \mathrm{~mm}$ in size) and post dilatation ranged 5.5-7.0 $\mathrm{mm}$ ). In the cases with successful recanalization three dedicated renal stents were implanted and three drug eluting coronary stents in the other cases. Stents were positioned to protrude 1-2 mm into the aortic lumen with an average inflation pressure of $12 \mathrm{~atm}$ for the implantation itself and reaching up to $20 \mathrm{~atm}$ for ostial trumpeting (or flaring).

Immediately after the stent deployment, a loading dose of clopidogrel $450 \mathrm{mg}$, followed by maintenance dose of $75 \mathrm{mg} /$ day was administered for at least 12 months, and aspirin at a dose of $100 \mathrm{mg} /$ day was administered to all stented patients. In all of the patients a Doppler assessment of the stent patency was performed the day after the procedure. Then, a follow-up ultrasound was scheduled at 30 days, and at 3 and 6 months 
after the procedure. Additionally, control 24-h ABPM and plasma renin activity were scheduled 30 days after the procedure to evaluate BP control and the relationship with the renin levels.

\section{Results}

Percutaneous recanalization of the chronic renal artery occlusion was attempted in seven patients. The treatment showed technical success in six cases and was completed with good immediate angiographic result with no residual stenosis and transstenotic gradient and restored peripheral sub segmental blood flow. All seven patients were thoroughly monitored for adverse clinical events (vascular access complications, plasma creatinine levels increase, myocardial infarction, unexpected drop or increase of the systemic BP, bleeding complications, stroke or other neurological events). No adverse clinical events were registered during the hospital stay and 30 days after the procedure.

The 24-h ABPM in the successfully recanalized renal CTO patients, performed 30 days after the renal stunting showed a dramatic decrease in the BP values, with an average of 132.5/81.7 mmHg compared to $167.1 / 95.1 \mathrm{mmHg}$ before the procedure. In the patient with unsuccessful recanalization, no significant change of BP control or medication was registered. In the successful recanalization group the medication intake dropped from average 5.11 agents per patient before the procedure to an average of 1.17 per patient. Two patients needed no medication for the arterial hypertension, two patients were using only one medication, one was on double therapy, and one on triple therapy. In all of the cases, adequate BP control was achieved with ABPM not exceeding values of 140/90 mmHg.

During the 6-month follow-up, two of the patients had instent restenosis with reoccurrence of the unsatisfactory BP control with registered re-elevation of the PRA. In the first case (the female patient with Takayasu arteritis), this occurred 4 months after the procedure, and in the second case it occurred after 3 months. Both patients underwent successful second PTA: in the first patient, a second stent was implanted and treatment with oral rapamycin $2 \mathrm{mg} /$ day was administered for 1 month to prevent reoccurrence of restenosis. The second patient was treated with efficient balloon PTA with a noncompliant balloon and additional drug eluting balloon inflation.

Further, the patients were followed up by Doppler ultrasound of the renal arteries and a clinical exam for an average of 18 months (6-26 months). All of the patients had normal BP values, $<130 / 85 \mathrm{mmHg}$ at the 12-month visit and no evidence of restenosis (secondary patency rate $=100 \%$ ). They followed their existing treatment regimen: two patients with no therapy and other two with monotherapy.

\section{Discussion}

The prevalence of renal artery stenosis in the elderly hypertensive patients approaches $20 \%$ after the age of 70 years
[12]. Approximately 5\% of renal artery lesions with $>60 \%$ diameter stenosis will progress to complete occlusion over a period of 3 years[13]. Critical renovascular disease is associated with substantial morbidity and mortality. The survival rate at 2 years is a dismal $56 \%$, with the majority of deaths associated with complications of vascular disease [14]. In several studies in patients undergoing renal dialysis, the prevalence of renal artery disease is $10-20 \%$ [15].

Acute pulmonary edema is not an infrequent presentation of severe renovascular disease in the elderly [10] and carries high risk in patients with dicreased cardiopulmonary reserve and is one of the current indications in the present guidelines. The DRASTIC, EMMA and ASPIRE-2 studies showed that renal stenting resulted in improvement of $\mathrm{BP}$ and reduced the number of antihypertensive medications [16,17]. The ASTRAL and CORAL studies showed that there is no significant difference between the group with renal stenting and the group medical therapy $[18,19]$.

Doppler ultrasonography may potentially have good accuracy in assessing renal artery stenosis/occlusion and preserved sub segmental collateral blood flow, and it may be a useful tool for assessing which patient will respond to endovascular revascularization in renal stenosis, but requires a well-trained operator and adequate ultrasound windows. It has been suggested that a resistive index $>0.8$ predicts poor response to revascularization [20]. Several small trials have tried to establish surgical treatment as a standard procedure to treat resistant hypertension in case of total renal occlusion. Whitehouse published the results of 30 patients with renal CTO surgically treated. The registered mortality was $6.6 \%$ and the nephrectomy incidence was 36.6\% [21].

Endovascular revascularization of occluded renal artery is still considered controversial, but has the potential of lower morbidity/mortality $[7,8]$. Total occlusion of renal arteries is not considered as a standard indication for endovascular intervention. There are no trials or big series published to date. We found several case descriptions with favorable results from the procedural and clinical point of view.

Murat Sezer etal. [10] reported two cases of revascularization of occluded renal artery in patients with severe hypertension and high level of creatinin. After the procedure, they established significant improvement over the control of BP with a significant reduction of used antihypertensive drugs [10].

Wykrzykowska et al. [11] reported a case of an 81-yearold woman with a history of giant-cell arteritis, hypertension, tobacco use, and peripheral vascular disease presented with acute hypoxic respiratory failure due to pulmonary edema that required mechanical ventilation and chronically occluded the left renal artery. After recanalizatoin of chronically occluded artery reported improvement in the patient's hypertension and congestive symptoms [11]. 
Yokoy et al. [22] reported a case of left renal subtotal stenosis and right renal artery total stenosis and concomitant CAD with favorable clinical result after bilateral renal artery intervention (better BP control and improved renal function) [22]. They suggested that preserved blood flow and kidney structure may be one of the factors indicating renal artery CTO recanalization. Our personal opinion is that preserved collateral flow to the kidney can lead to functional survival of the juxtaglomerular apparatus; thereby leading to high renin production and resistant hypertension totally independent from kidney's functioned and size. On the other hand, the preserved collateral flow is, at the same time, a predictor of efficient BP control after successful recanalization. In our cases, high level of rennin production together with preserved sub segmental collateral flow was an indication for intervention and a predictor for clinical success, and both are considered as possible predictors for significant reduction of $\mathrm{BP}$ values after successful recanalization. This has to be proven in larger trials.

Vascular access and French size of the materials used for renal artery interventions is another interesting point of discussion. According to our extensive experience with renal artery interventions (including not only PTA and stunting but also renal denervation in more than 200 patients during the last 5 years) upper access (radial or brachial) is more convenient for renal interventions because in hypertensive subjects, the renal artery arises at a sharp angle from the aorta in the caudal direction causing complications for the manipulation of catheters and devices from the "classic" femoral access in many cases. In these cases, is much easier and faster to engage a guiding catheter (in our protocol the JR 4.0/ 6 Fr is the preferred one) into the ostium of the renal artery or at least to be axial versus the axis of the renal artery (because in part of the cases the occlusion is almost strictly ostial and real engagement is not possible).

In these cases, crossing across the minimal proximal stump is much more probable because of the better push ability of devices such as micro catheters and wires and better axial alignment between the axis of the guiding catheter and the axis of the occluded renal artery. It also provides better support for driving and implanting a stent. Other reason to use a preferably radial approach is the fact that it is related to significantly lower incidence of bleeding complications and lower overall MACE that is categorically shown in several "coronary" trials (such as RIVAL and RIFLE) [23].

Regarding the French size: nowadays major part of contemporary interventional devices including stent systems dedicated to visceral and renal arteries are compatible with 6 Fr guiding catheters. In many cases, the size of the kidney and the artery in case of CTO are smaller than the usual, advocating the use of coronary DES in order to prevent restenosis and enabling the use of $6 \mathrm{FR}$, thus allowing really minimally invasive approach (radial/ulnar) and reducing vascular complications and morbidity and mortality.

Size of the treated kidney in our series the average size of the treated kidney $(6.3 \mathrm{~cm})$ is much smaller compared to the indicated size approved in the guidelines for intervention. The reason we did not consider the contraindication for intervention in such "small" kidneys is because we were studying the decrease in rennin level and not improvement of kidney function. In some of our patients, the excretion function of such kidneys was categorically shown (by nuclear investigation) to be totally lost and was less probable to be restored after recanalization. However, as shown in our group of patients, the kidney size does not affect the potential for high rennin production and the potential for beneficial effect on the BP control after successful recanalization and normal systemic flow restoration reaching the receptors in the juxtaglomerular apparatus (negative feedback mechanism).

\section{Conclusion}

In case of renal occlusion and resistant $\mathrm{AH}$, preserved renin production as a consequence of preserved collateral subsegmental blood flow is a predictor of clinical success after recanalization. The most probable mechanism of $\mathrm{AH}$ is the preserved vascular microcirculation allowing juxtaglomerular survival and elevated renin production. At the same time, preserved micro circular perfusion is a predictor of lowering renin levels and success of BP control after renal СTO recanalization. There is evidence of a direct relationship between preserved parenchyma flow and expected post interventional result in terms of BP control, confirmed in our cases.

The recanalization of total renal artery occlusions is feasible and safe and has a positive effect on lowering the plasma renin activity and BP control. This procedure has to be applied only to a selective group of patients with resistant hypertension and evidence of preserved sub segmental flow. Further, larger multicentre trials need to be performed to establish the exact indications and results before broader application of this strategy to the daily clinical practice.

\section{References}

1. Kearney PM, Whelton M, Reynolds K, Muntner P, Whelton PK, et al. (2005)Global burden of hypertension: analysis of worldwide data. Lancet 365(9455): 217-223.

2. Spitalewitz S, Reiser IW (1996) Atherosclerotic renovascular disease. Am J Ther 3(4): 21-28.

3. Robert D Safian, Stephen C Textor (2001) Renal artery stenosis. N Engl J Med 344(6): 431-442.

4. Dorros G, Jaff M, Mathiak L, Dorros II, Lowe A, et al. (1998) Four-year follow-up of Palmaz-Schatz stent revascularization as treatment for atherosclerotic renal artery stenosis. Circulation 98(7): 642- 647.

5. Gruntzig A, Kuhlmann U, Vetter W, Lütolf U, Meier B, et al. (1978) Treatment of renovascularhypertension with percutaneous transluminal dilatation of a renalarterystenosis. Lancet 1(8068): 801802 . 
6. Gruntzig A (1978) Transluminal dilatation of coronary-artery stenosis Lancet 1(8058): 263.

7. Hirsch AT, Haskal ZJ, Hertzer NR, Bakal CW, Creager MA, et al. (2006) ACC/AHA 2005 Practice Guidelines for the management of patients with peripheral arterial disease (lower extremity, renal, mesenteric, and abdominal aortic): a collaborative report from the American Association for Vascular Surgery/Society for Vascular Surgery, Society for Cardiovascular Angiography and Interventions, Society for Vascular Medicine and Biology, Society of Interventional Radiology, and the ACC/ AHA Task Force on Practice Guidelines (Writing Committee to Develop Guidelines for the Management of Patients With Peripheral Arteria Disease): endorsed by the American Association of Cardiovascular and Pulmonary Rehabilitation; National Heart, Lung, and Blood Institute; Society for Vascular Nursing; TransAtlantic Inter-Society Consensus; and Vascular Disease Foundation. Circulation 113(11): e463-e654.

8. Parikh SA, Shishehbor MH, Gray BH, White CJ, Jaff MR (2014) SCAI expert consensus statement for renal artery stenting appropriate use. Cathet Cardiovasc Interv 84(7): 1163-1171.

9. van Jaarsveld BC, Krijnen P, Pieterman H, Derkx FH, Deinum J, et al. (2000)The effect of balloon angioplasty on hypertension in atherosclerotic renal-artery stenosis.Dutch Renal Artery Stenosis Intervention Cooperative Study Group. N Engl J Med 342(14): 1007 1014.

10. Pickering TG, Herman L, Devereux RB, Sotelo JE, James GD, et al. (1988) Recurrent pulmonary oedema in hypertension due to bilateral renal artery stenosis: Treatment by angioplasty or surgical revascularisation. Lancet 2(8610): 551-552.

11. Sezer M, Nisanci Y, Ozsaruhan O, Olcay A, Ecder T (2003)Stenting for bilateral renal artery occlusion: a report of two cases. Nephrol Dial Transplant 18(12): 2663-2664.

12. Coen G, Calabria S, Lai S, Moscaritolo E, Nofroni I, et al. (2003) Atherosclerotic ischemic renal disease. Diagnosis and prevalence in an hypertensive and/or uremic elderly population. BMC Nephrol 4: 2 .

13. Caps MT, Zierler RE, Polissar NL, Bergelin RO, Beach KW, et al. (1998) Risk of atrophy in kidneys with atherosclerotic renal artery stenosis. Kidney Int 53(3): 735-742.
14. Eggers PW, Connerton R, McMullan M (1984) The Medicare experience with end-stage renal disease: Trends in incidence, prevalence, and survival. Health Care Financ Rev 5(3): 69-88.

15. Scoble JE, Maher ER, Hamilton G, Dick R, Sweny P, et al. (1989) Atherosclerotic renovascular disease causing renal impairment -A case for treatment. Clin Nephrol 31(3): 119-122.

16. Rocha-Singh K, Jaff MR, Rosenfield K (2005)Evaluation of the safety and effectiveness of renal artery stenting after unsuccessful balloon angioplasty: the ASPIRE-2 study. J Am Coll Cardiol 46(5): 776-783.

17. van Jaarsveld BC, Krijnen P, Pieterman H, Derkx FH, Deinum J, et al. (2000)The effect of balloon angioplasty on hypertension in atherosclerotic renal-artery stenosis.Dutch Renal Artery Stenosis Intervention Cooperative Study Group. N Engl J Med 342(14): 10071014.

18. ASTRAL Investigators, Wheatley K, Ives N, Gray R, Kalra PA, et al. (2009)Revascularization versus medical therapy for renal-artery stenosis. N Engl J Med 361(20): 1953-1962.

19. Cooper CJ, Murphy TP, Cutlip DE, Jamerson K, Henrich W, et al. (2014) Stenting and medical therapy for atherosclerotic renal-artery stenosis. N Engl J Med 370(1): 13-22.

20. Radermacher J, Chavan A, Bleck J, Vitzthum A, Stoess B, et al. (2001) Use of Doppler Ultrasonography to Predict the Outcome of Therapy for Renal-Artery Stenosis. N Engl J Med 344(6): 410-417.

21. Whitehouse WM, Kazmers A, Zelenock GB, Erlandson EE, Cronenwett JL, et al. (1981) Chronic total renal artery occlusion: effects of treatment on secondary hypertension and renal function. Surgery 89(6): 753-763.

22. Yokoy, Kawarada (2007) Recanalization of Renal Artery CTOs. Is it worth renal artery occlusion? Endovascular Today 50-52.

23. Cantor WJ, Mehta SR, Yuan F, Džavík V, Worthley M, et al. (2015) Radial versus femoral access for elderly patients with acute coronary syndrome undergoing coronary angiography and intervention: insights from the RIVAL trial. Am Heart J 170(5): 880-886.

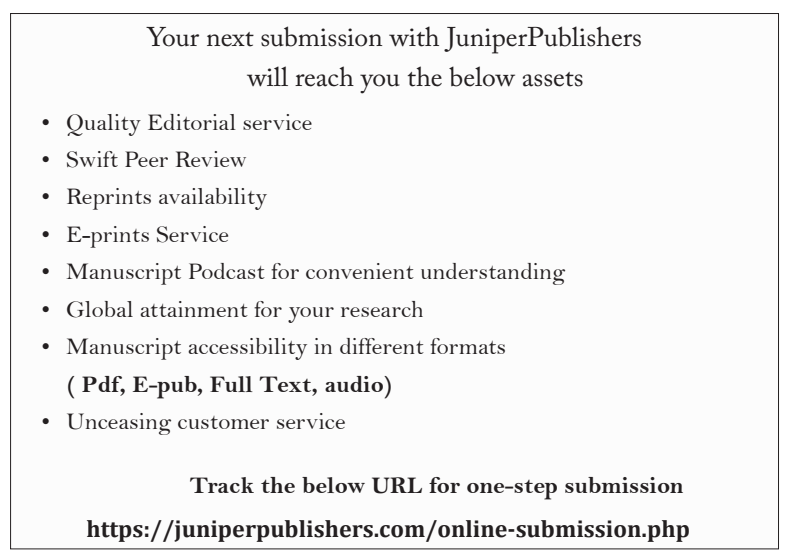

